Case Report

\title{
Magnetic Resonance Imaging and Flexible Hysterofiberscopic Findings of a Uterine Adenofibroma: Case Report and Literature Review
}

\author{
Hideki Watanabe, Naoya Harada (D), Ichiro Nobuhara, Noriko Haruta, \\ Yumi Higashiura, and Shioka Watanabe \\ Department of Obstetrics and Gynecology, Nara City Hospital, 1-50-1 Higashikidera-cho, Nara City, Nara Prefecture 630-8305, Japan \\ Correspondence should be addressed to Naoya Harada; harada@nara-jadecom.jp
}

Received 12 December 2017; Accepted 9 January 2018; Published 31 January 2018

Academic Editor: Giampiero Capobianco

Copyright ( 2018 Hideki Watanabe et al. This is an open access article distributed under the Creative Commons Attribution License, which permits unrestricted use, distribution, and reproduction in any medium, provided the original work is properly cited.

To our knowledge, highly detailed findings of flexible hysterofiberscopy in patients with adenofibroma have not been described. A 75-year-old nulliparous asymptomatic woman presented with a uterine polyp, which exhibited punctate heterogeneous hyperintensity or islands of isointense-to-hypointense signals on T2-weighted magnetic resonance imaging (MRI), hypointense signals on T1-weighted images (T1WI), and a little enhancement on contrast-enhanced T1WI. Flexible hysterofiberscopy revealed a red-pink polyp with a white-yellow, cobblestone-like surface easily deformed by perfusion fluid. The tumor was diagnosed histologically as an adenofibroma. Total abdominal hysterectomy and bilateral salpingo-oophorectomy were performed. The tumor in the uterus was necrotic macroscopically and histologically, and a residual adenofibroma could not be confirmed. At present, two years after surgery, the patient remains healthy. MRI and hysterofiberscopy can reveal the histological features of uterine adenofibromas and be useful for their diagnosis.

\section{Introduction}

Adenofibroma is an extremely rare benign biphasic neoplasm classified as an epithelial and mesenchymal tumor. Only approximately 30 cases have been reported to date (Table 1) [1-18]. Attempts have been made to diagnose these tumors using ultrasonography, computed tomography (CT), magnetic resonance imaging (MRI), hysteroscopy, and/or biopsy, but these methods were successful in correctly identifying adenofibroma in only three patients $[3,16,17]$. To date, only three patients each were assessed using MRI $[11,12,14]$ and hysteroscopy $[5,15,16]$. We encountered a patient diagnosed histologically with adenofibroma using MRI and flexible hysterofiberscopy. To our knowledge, this is the first patient with adenofibroma who was assessed using both MRI and hysterofiberscopy. Highly detailed hysterofiberscopic findings in adenofibroma have not been reported previously. This report describes the clinical characteristics of this patient, including findings on MRI and flexible hysterofiberscopy, and provides a literature review of the clinical features of this rare neoplasm.

\section{Case Report}

A 75-year-old nulliparous woman was referred to our hospital by a private internal medicine clinic for an asymptomatic intrauterine mass. She had been treated for type 2 diabetes mellitus and cholelithiasis for 20 years. The mass was detected on abdominal ultrasonography at the clinic. Transvaginal color Doppler ultrasonography at our hospital revealed a polypoid mass, measuring $3 \times 2 \mathrm{~cm}$, in the uterine cavity, and consisting of multiple low echogenic cysts that differed in size without pulsatile blood flow (Figure 1). The patient's serum CA125, CA19-9, CEA, SCC, and LDH concentrations were $11.2 \mathrm{U} / \mathrm{mL}, 6.7 \mathrm{U} / \mathrm{mL}, 2.7 \mathrm{ng} / \mathrm{mL}, 1.0 \mathrm{ng} / \mathrm{mL}$, and $259 \mathrm{IU} / \mathrm{mL}$, respectively. MRI also detected an intrauterine tumor, which exhibited punctate heterogeneous hyperintensity or islands of isointense-to-hypointense signals on T2-weighted images (T2WI) (Figures 2(a) and 2(b)). Axial T1-weighted images 


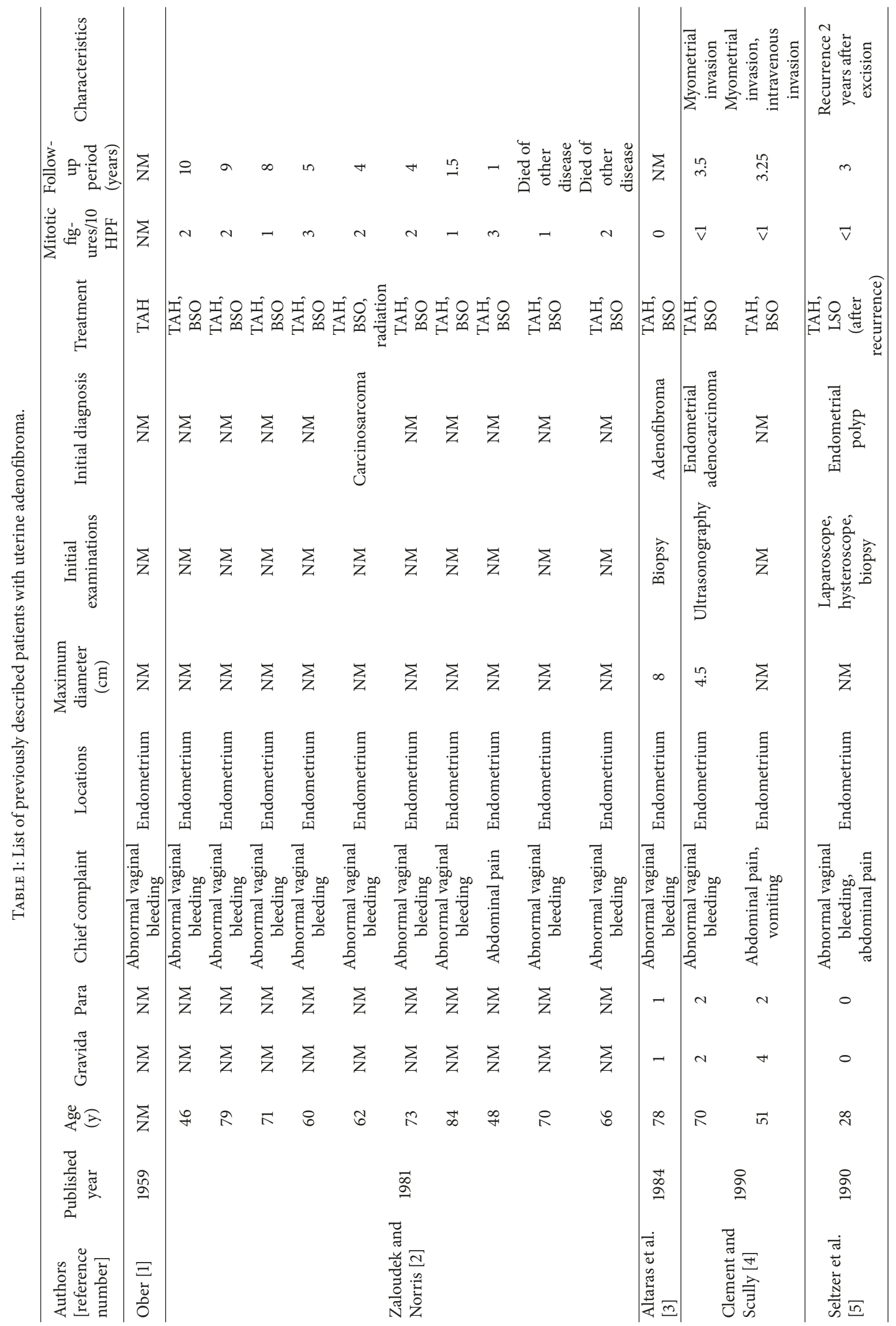




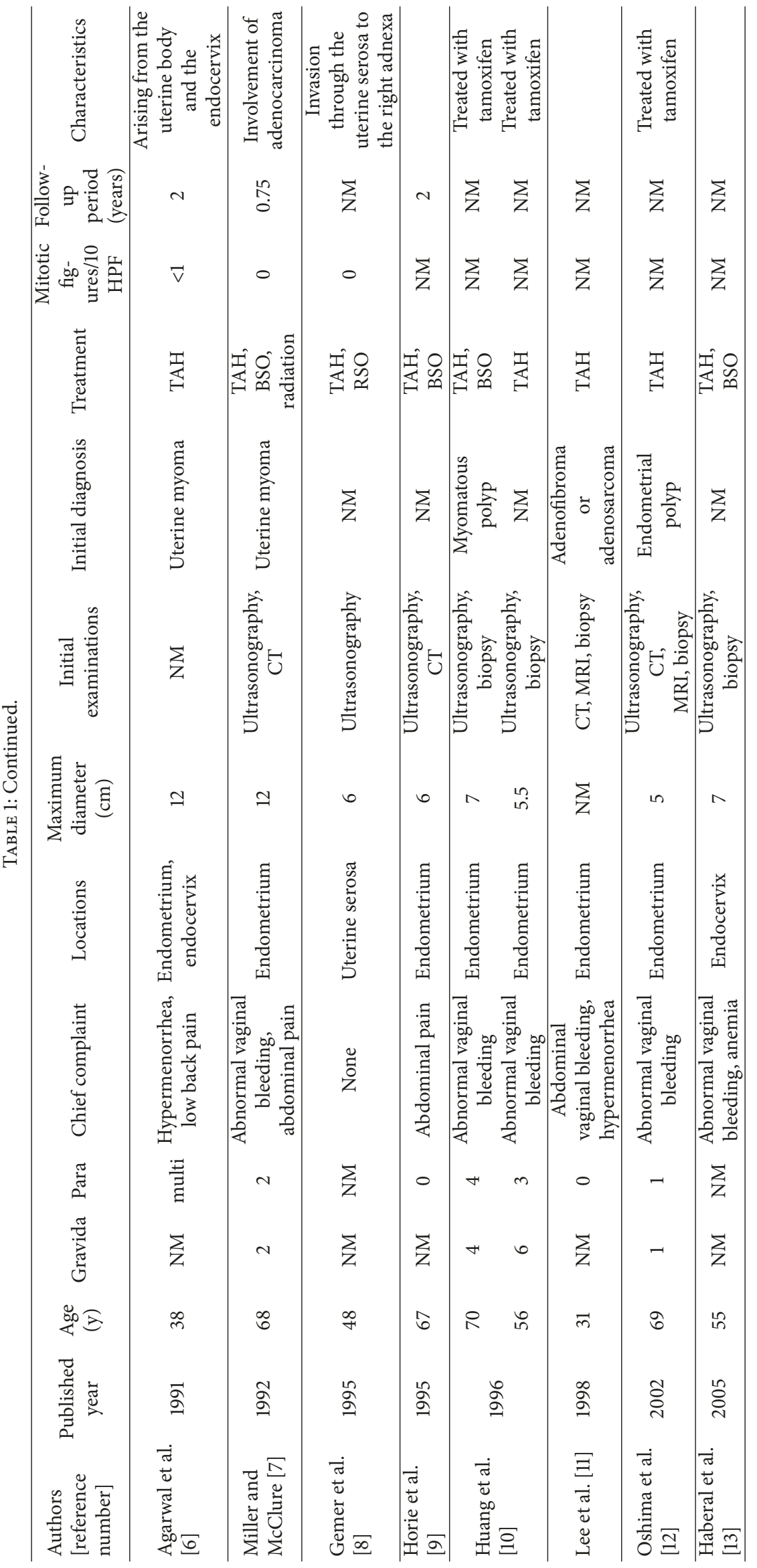




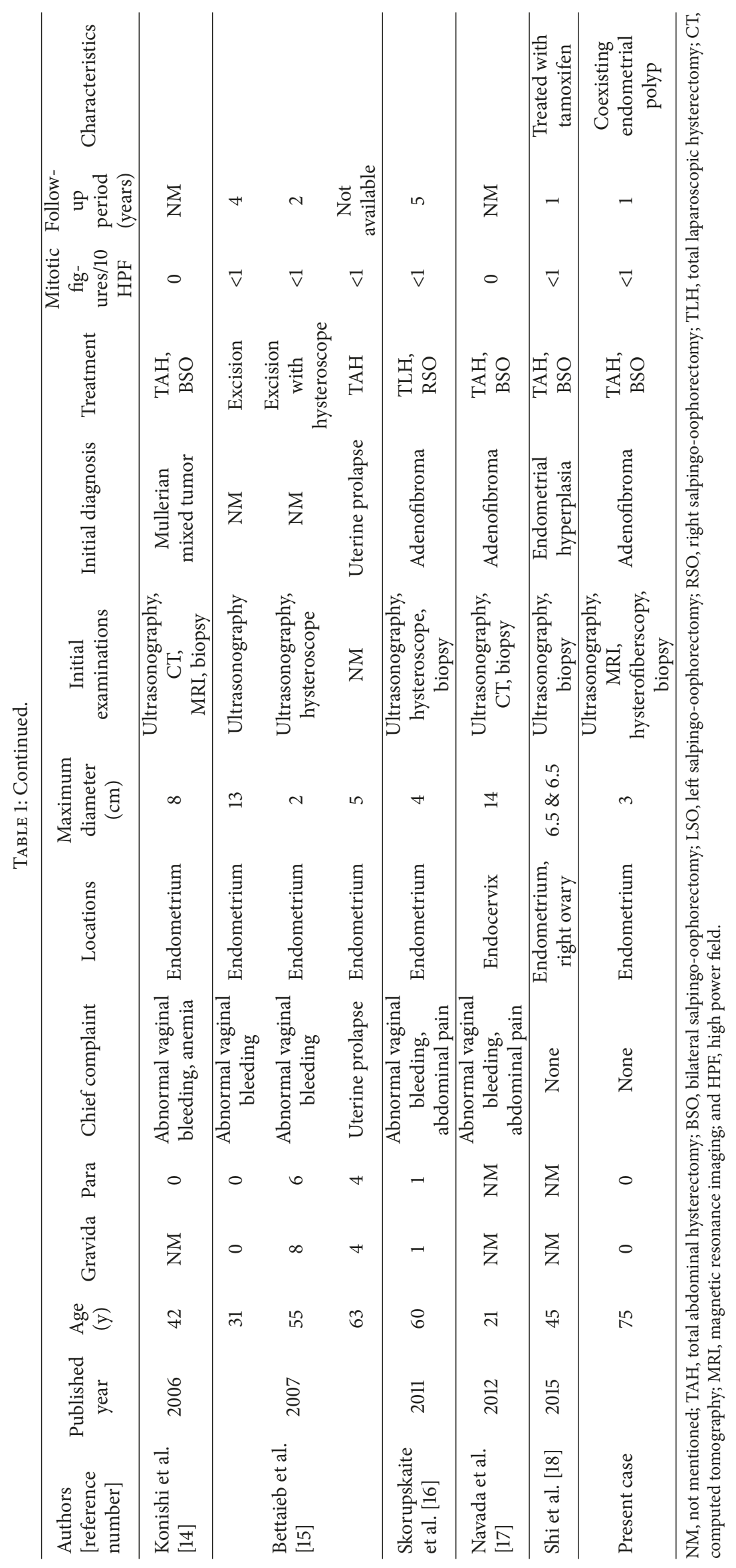




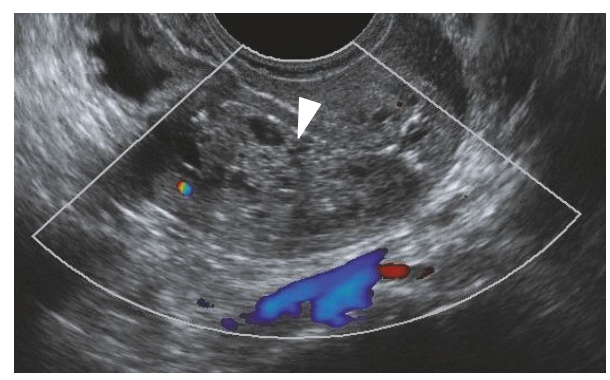

FIgURE 1: Figures on transvaginal color Doppler ultrasonography, showing a mass in the uterine cavity, measuring $3 \times 2 \mathrm{~cm}$ and consisting of multiple cysts differing in size (triangle) and without pulsatile blood flow.

(T1WI) showed a hypointense signal with focal areas of high signal intensity, suspected of being hemorrhagic foci (Figure 2(c)), whereas axial contrast-enhanced T1WI showed insignificant enhancement (Figure 2(d)). The tumor was well-circumscribed without myometrial invasion, with no high-intensity areas on diffusion-weighted images. Flexible hysterofiberscopy revealed a reddish-pink polyp with a whitish-yellow, cobblestone-like surface (Figure 3(a)), easily deformed by perfusion fluid (Figure 3(b)). Transcervical exeresis was attempted using forceps, but only a small part of the tumor was removed because the cervix was insufficiently dilated. Microscopically, the tumor consisted of a benign biphasic proliferation of epithelial and mesenchymal components (Figure 4(a)). The epithelial elements were endometrial glands of benign appearance. The mesenchymal component was an endometrial stroma containing fibroblasts of benign nuclear features and very low mitotic activity. The mesenchymal part was strongly positive on Masson's trichrome staining, confirming the presence of collagen fibers (Figure 4(b)). Immunohistochemical staining showed that the stromal cells were negative for smooth muscle actin and CD10. These features suggested that the tumor was an adenofibroma. Hysterectomy was recommended, because an adenosarcoma may be present within the residual tumor or an adenofibroma may develop invasive potential and recur $[4,5]$. Therefore, total abdominal hysterectomy and bilateral salpingo-oophorectomy were performed. Macroscopically, the mass in the uterus measured approximately $4 \times 2.5 \mathrm{~cm}$ and was a gray-semitransparent-edematous necrotic tumor (Figure 5), although the attached area could not be identified. Histological examination revealed that the tumor was completely necrotic and amorphous, such that its origin could not be determined. Residual adenofibroma could not be confirmed in the uterus. One part of the endometrium contained an endometrial polyp, with a pathological morphology that differed completely from the adenofibroma. The postoperative course of the patient was uneventful, and no further treatment was required. Currently, two years after the surgery, the patient remains healthy.

\section{Discussion}

Endometrial adenofibroma was first described in 1959 as a benign form of mixed mesodermal tumor [1]. This rare type of noninvasive neoplasm is composed of benign epithelial and mesenchymal components, which can usually be sharply delineated from the underlying myometrium and adjacent endometrium. A similar type of tumor, Mullerian adenosarcoma, was first described in 1974 [19]. Mullerian adenosarcoma is a mixed mesodermal tumor in which the epithelium is benign, but the stromal component is histologically sarcomatous [19]. It is important to distinguish adenofibroma from adenosarcoma, as their expected clinical behavior differs. The most useful criterion for distinguishing adenofibroma from adenosarcoma is the frequency of mitotic figures in the stroma, with adenofibromas having three or fewer mitotic figures per 10 high-power fields (HPFs) and adenosarcomas having four or more mitotic figures per $10 \mathrm{HPFs}$ [2]. Moreover, in contrast to adenosarcomas, adenofibromas do not have a marked degree of atypical mesenchymal cells, a histologically malignant heterologous element, or myometrial invasion [2]. However, one study described two adenofibromas that infiltrated deep into the myometrium, with one invading the lumen of myometrial veins [4]. The latter case resembles intravenous leiomyomatosis, a histologically benign leiomyoma derived from a uterine leiomyoma or intrauterine venous wall that grows and extends intravenously. The origin and malignant potential of these tumors remain unclear.

To date, approximately 30 cases of adenofibroma have been reported in the literature (considering only English publications) (Table 1) [1-18]. Adenofibromas occur primarily in postmenopausal women, of an average age of 57 years, but may also occur in women of reproductive age [17]. The chief complaints are usually abnormal vaginal bleeding and/or abdominal pain. About $90 \%$ of adenofibromas arise in the endometrium, with the other $10 \%$ reported to originate from the uterine endocervix. These tumors range in size from 2 to $14 \mathrm{~cm}$, with an average diameter of $7.1 \mathrm{~cm}$. Adenofibroma has been associated with tamoxifen therapy for breast cancer [10, $12,18]$. Tamoxifen is a selective estrogen receptor modulator widely used to treat patients with estrogen-dependent breast cancer. Tamoxifen is thought to act as a partial estrogen agonist on the endometrium, thereby increasing the incidence of proliferative endometrial lesions, including adenofibromas, polyp, endometrial hyperplasia, and endometrioid adenocarcinomas.

Although attempts have been made to diagnose adenofibroma using ultrasonography, CT, MRI, hysteroscopy, and/or biopsy, these methods were successful in only three patients $[3,16,17]$. To date, only three patients each have been evaluated using MRI $[11,12,14]$ and hysteroscopy $[5,15,16]$. To our knowledge, this is the first patient who was diagnosed using both MRI and hysteroscopy. MRI findings in this patient were consistent with those previously described [11, $12,14]$. However, to our knowledge, highly detailed findings of flexible hysterofiberscopy have not been reported previously in patients with adenofibroma. Our findings of cobblestonelike surface and easy deformation by perfusion fluid reflect the histological features of adenofibroma with multilocular cysts containing secreted fluid.

Although endometrial adenofibromas are benign lesions, total hysterectomy is recommended, because these 


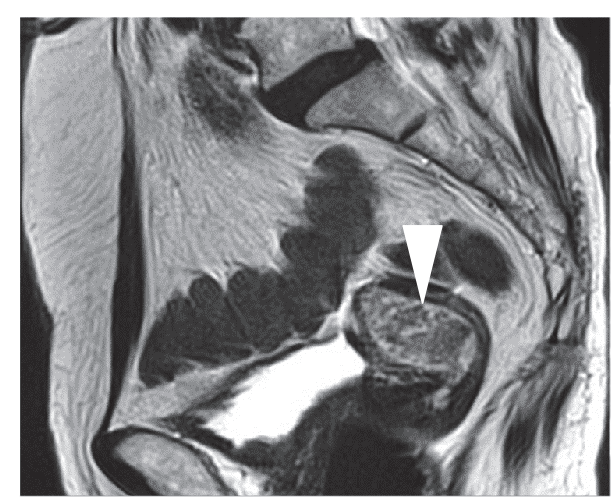

(a)

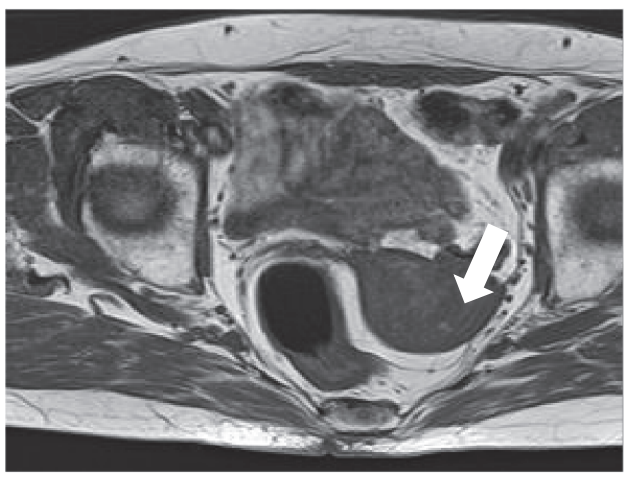

(c)

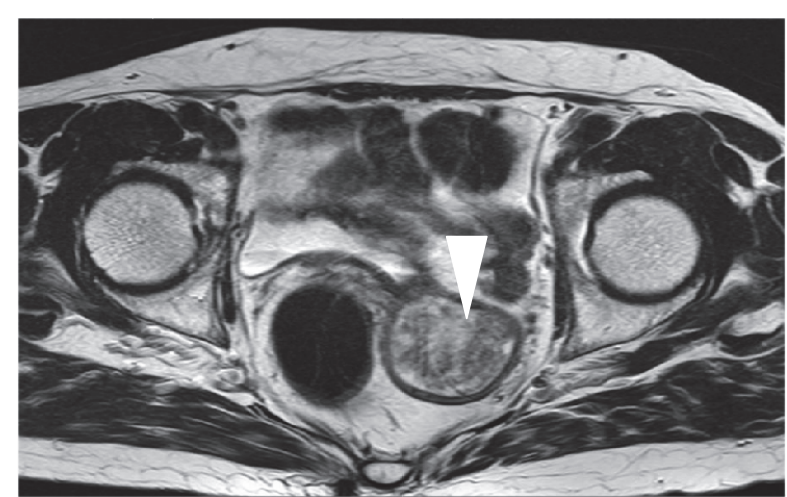

(b)

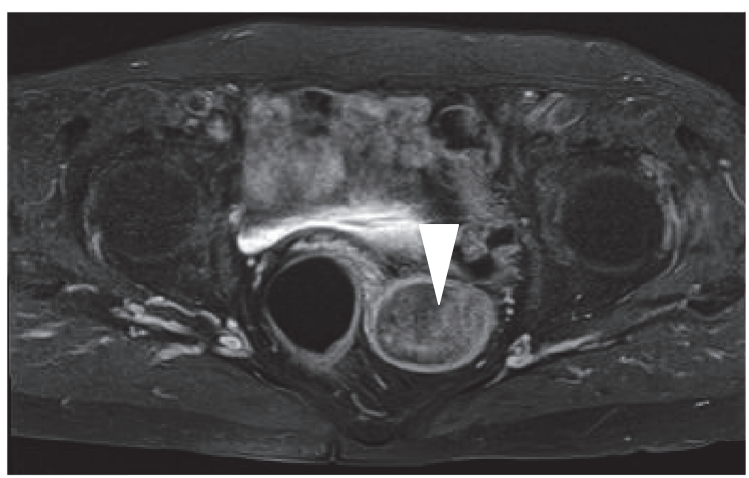

(d)

FIGURE 2: Magnetic resonance imaging of this patient. (a) Sagittal T2-weighted images (T2WI), showing an intrauterine tumor (triangle), which exhibited punctate heterogeneous hyperintensity or islands of isointense-to-hypointense signals. (b) View of the tumor (triangle) on axial T2WI. (c) Axial T1-weighted images (T1WI), showing that the tumor was detected as a hypointense signal with focal areas of high signal intensity (arrow), suspected of being hemorrhagic foci. (d) Axial contrast-enhanced T1WI showing a little tumor enhancement (triangle).

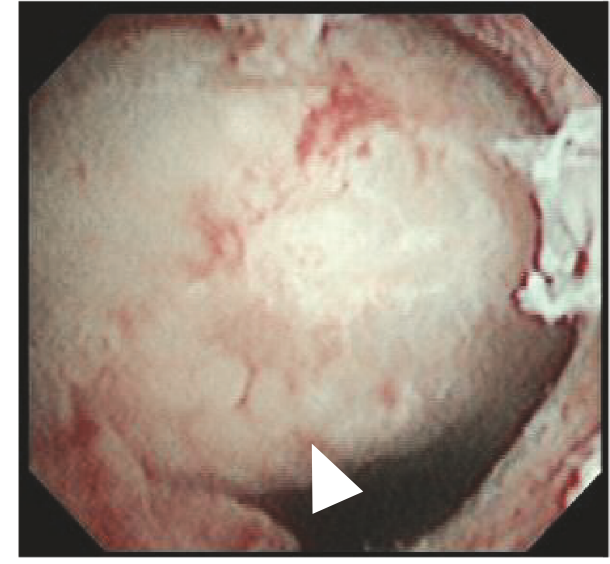

(a)

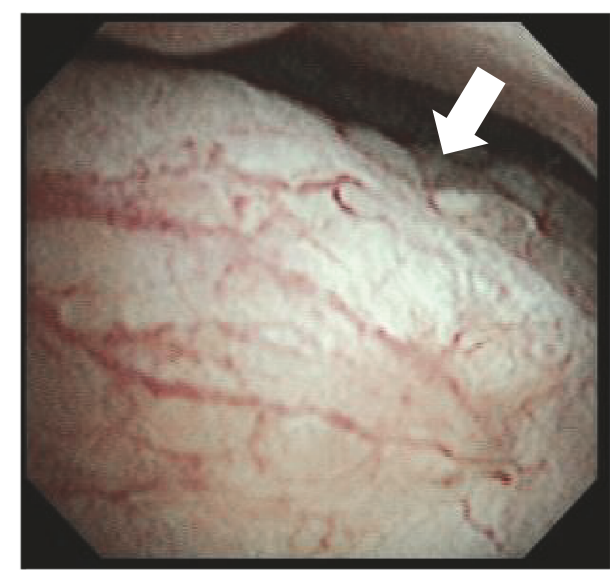

(b)

FIGURE 3: Hysterofiberscopy findings in this patient, showing (a) a red-yellow-white polyp with a partially reticulated surface (triangle) in the uterine cavity; (b) the lesion was easily deformed by perfusion fluid (arrow).

neoplasms may recur if incompletely curetted or locally excised [4, 5]. Hysterectomy assures complete excision, as well as permitting the thorough sampling needed to exclude the possibility of adenosarcoma. Indeed, most patients with adenofibroma underwent hysterectomy, with none showing tumor recurrence $[1-14,16-18]$.
Young women with adenofibroma may be given the option of lesion removal under hysteroscopic visualization, allowing retention of the uterus and reproductive potential. Surgical excision of adenofibromas in two patients, including one who underwent operative hysteroscopy with wide local excision, was successful, with no evidence of 


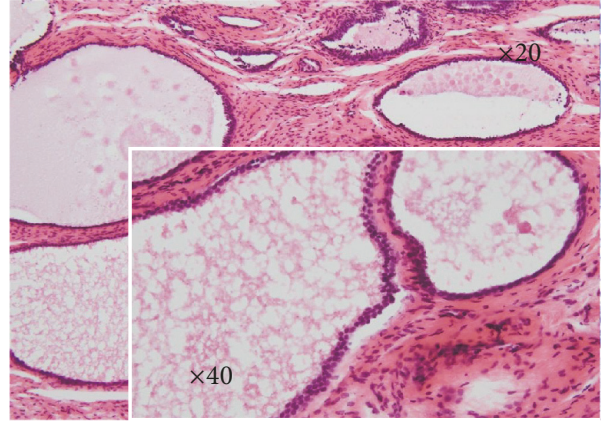

(a)

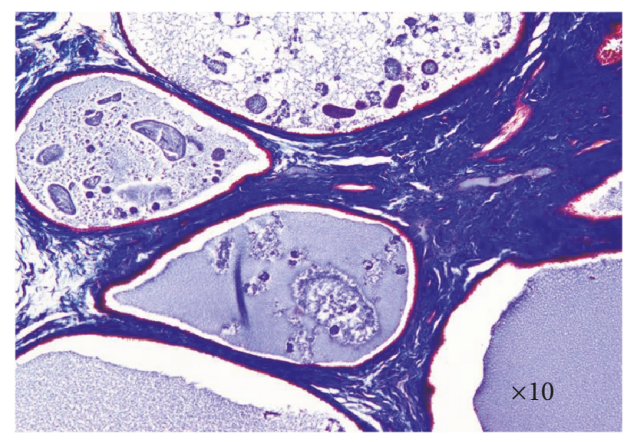

(b)

Figure 4: Microscopic findings, showing that (a) the tumor resulted from the benign biphasic proliferation of epithelial and mesenchymal components. The epithelial elements were endometrial glands of benign appearance (HE stain, objective magnification $\times 20$ ). The inset shows that the mesenchymal component consisted of endometrial stroma containing fibroblasts of benign nuclear features and very low mitotic activity (HE stain, objective magnification $\times 40$ ): (b) the mesenchymal part was strongly positive on Masson's trichrome staining, confirming the presence of collagen fibers (Masson's trichrome stain, objective magnification $\times 10$ ).

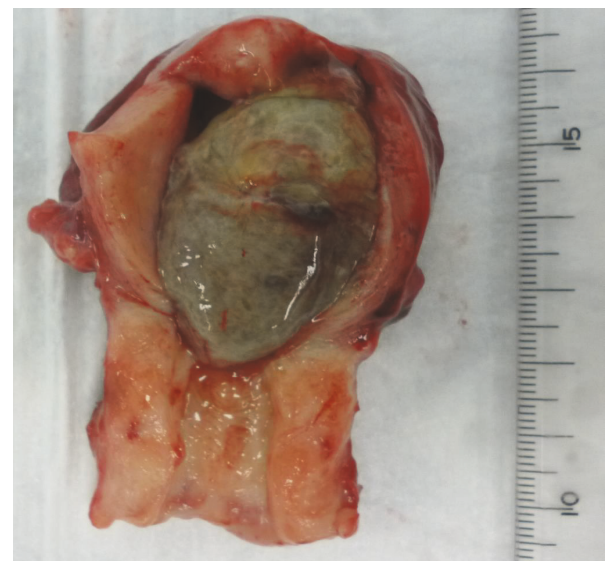

FIgURE 5: Macroscopic findings, showing a gray-semitransparentedematous necrotic tumor, measuring approximately $4 \times 2.5 \mathrm{~cm}$, in the uterine cavity. The attached area could not be identified.

recurrence [15]. Recurrence associated with conservative treatment may be due to incomplete excision. Operative hysteroscopy with wide local excision may be considered an alternative to hysterectomy for women, who wish to preserve their reproductive function, provided that the completeness of excision is verified and long-term follow-up is possible.

The tumor in our patient was well demarcated, with no evidence of invasion into the myometrium on MRI. Necrotic tissue, likely the adenofibroma, was present in the uterine cavity after hysterectomy. Transcervical exeresis may have caused twisting of the tumor, resulting in necrosis. The tumor could be completely removed after hysterofiberscopy. The uterus did not contain any residual adenofibroma tissue, but the endometrium contained an endometrial polyp. Because the pathological morphology of these tumors differed completely, their causal relationship could not be determined.

\section{Conclusion}

Flexible hysterofiberscopy and MRI are also useful for the diagnosis of adenofibroma, which formed a polypoid mass in the uterine cavity.

\section{Conflicts of Interest}

All authors declare that there are no conflicts of interest regarding the publication of this paper.

\section{References}

[1] W. B. Ober, "Uterine sarcomas: histogenesis and taxonomy," Annals of the New York Academy of Sciences, vol. 75, no. 2, pp. 568-585, 1959.

[2] C. J. Zaloudek and H. J. Norris, "Adenofibroma and adenosarcoma of the uterus: a clinicopathologic study of 35 cases," Cancer, vol. 48, no. 2, pp. 354-366, 1981.

[3] M. Altaras, I. Cohen, M. Cordoba, and N. Ben Aderet, "Papillary adenofibroma of the endometrium: Case report and review of the literature," Gynecologic Oncology, vol. 19, no. 2, pp. 216-221, 1984.

[4] P. B. Clement and R. E. Scully, "Müllerian adenofibroma of the uterus with invasion of myometrium and pelvic veins," International Journal of Gynecological Pathology, vol. 9, no. 4, pp. 363-371, 1990.

[5] V. L. Seltzer, A. Levine, G. Spiegel, D. Rosenfeld, and E. L. Coffey, "Adenofibroma of the uterus: Multiple recurrences following wide local excision," Gynecologic Oncology, vol. 37, no. 3, pp. 427-431, 1990.

[6] P. K. Agarwal, N. Husain, and Chandrawati, "Adenofibroma of uterus and endocervix," Histopathology, vol. 18, no. 1, pp. 79-80, 1991.

[7] K. N. Miller and S. P. McClure, "Papillary adenofibroma of the uterus: Report of a case involved by adenocarcinoma and review of the literature," American Journal of Clinical Pathology, vol. 97, no. 6, pp. 806-809, 1992. 
[8] O. Gemer, C. Mor, and S. Segal, "Uterine adenofibroma presenting as a cystic adnexal mass," Archives of Gynecology and Obstetrics, vol. 256, no. 2, pp. 99-101, 1995.

[9] Y. Horie, S. Ikawa, K. Kadowaki, Y. Minagawa, J. Kigawa, and N. Terakawa, "Lipoadenofibroma of the uterine corpus. Report of a new variant of adenofibroma (benign müllerian mixed tumor)," Archives of Pathology \& Laboratory Medicine, vol. 119, no. 3, pp. 274-276, 1995.

[10] K.-T. Huang, C.-A. Chen, W.-F. Cheng et al., "Sonographic characteristics of adenofibroma of the endometrium following tamoxifen therapy for breast cancer: Two case reports," Ultrasound in Obstetrics \& Gynecology, vol. 7, no. 5, pp. 363-366, 1996.

[11] H. K. Lee, S. H. Kim, J. Y. Cho, and K. M. Yeon, "Uterine adenofibroma and adenosarcoma: CT and MR findings," Journal of Computer Assisted Tomography, vol. 22, no. 2, pp. 314-316, 1998.

[12] H. Oshima, H. Miyagawa, Y. Sato et al., "Adenofibroma of the endometrium after tamoxifen therapy for breast cancer: MR findings," Abdominal Imaging, vol. 27, no. 5, pp. 592-594, 2002.

[13] A. Haberal, A. P. Cil, M. Gunes, and D. Cavusoglu, "Papillary adenofibroma of the cervix: A case report," Ultrasound in Obstetrics \& Gynecology, vol. 26, no. 2, pp. 186-187, 2005.

[14] Y. Konishi, H. Sato, T. Fujimoto, H. Tanaka, O. Takahashi, and T. Tanaka, "Adenofibroma of the endometrium protruding into the vaginal cavity: Findings on transvaginal ultrasonography, MRI and CT,' Journal of Obstetrics and Gynaecology Research, vol. 32, no. 6, pp. 623-627, 2006.

[15] I. Bettaieb, A. Mekni, K. Bellil et al., "Endometrial adenofibroma: A rare entity," Archives of Gynecology and Obstetrics, vol. 275, no. 3, pp. 191-193, 2007.

[16] K. Skorupskaite, A. Al-Nafussi, and G. McKillop, "Diagnostic challenges in a rare case of müllerian adenofibroma of the uterus: Instructive case and literature review," Diagnostic Histopathology, vol. 17, no. 12, pp. 557-561, 2011.

[17] H. M. Navada, B. P. Bhat, G. Ramani, R. R. Gatty, and C. S. Jayaprakash, "Unusual presentation of rare case of papillary adenofibroma of cervix in a young woman," Case Reports in Oncological Medicine, vol. 2012, pp. 1-3, 2012.

[18] H. Shi, X. Chen, B. Lv, and X. Zhang, "Concurrent tamoxifenrelated Müllerian adenofibromas in uterus and ovary," International Journal of Clinical and Experimental Pathology, vol. 8, no. 11, pp. 15381-15385, 2015.

[19] P. B. Clement and R. E. Scully, "Mullerian adenosarcoma of the uterus. A clinicopathologic analysis of ten cases of a distinctive type of mullerian mixed tumor," Cancer, vol. 34, no. 4, pp. 11381149, 1974. 


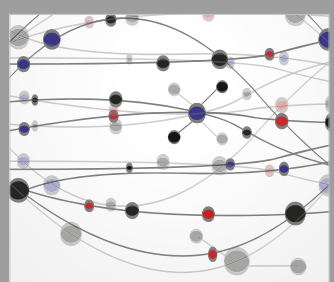

The Scientific World Journal
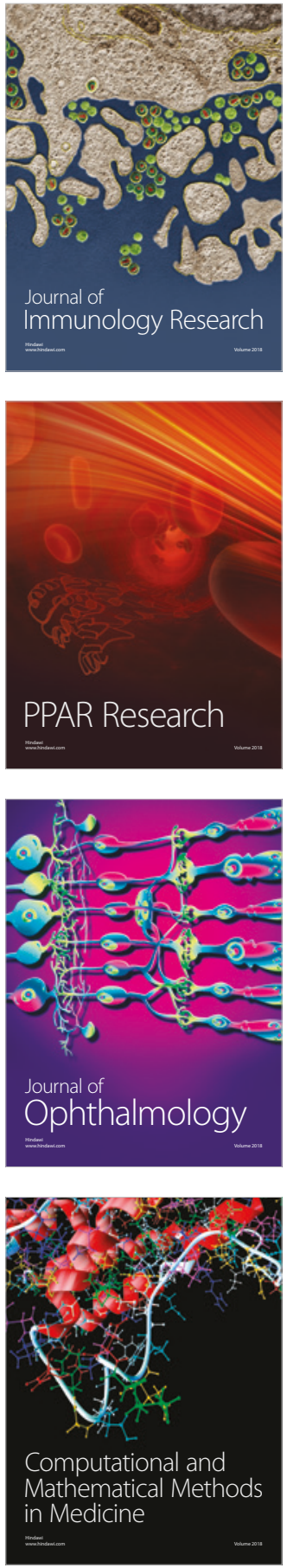

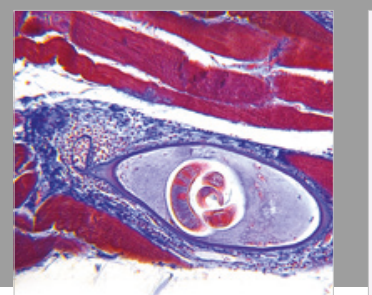

Gastroenterology Research and Practice

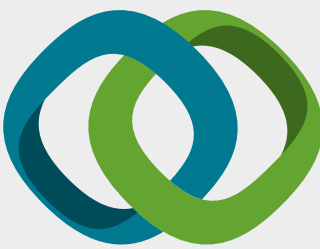

\section{Hindawi}

Submit your manuscripts at

www.hindawi.com
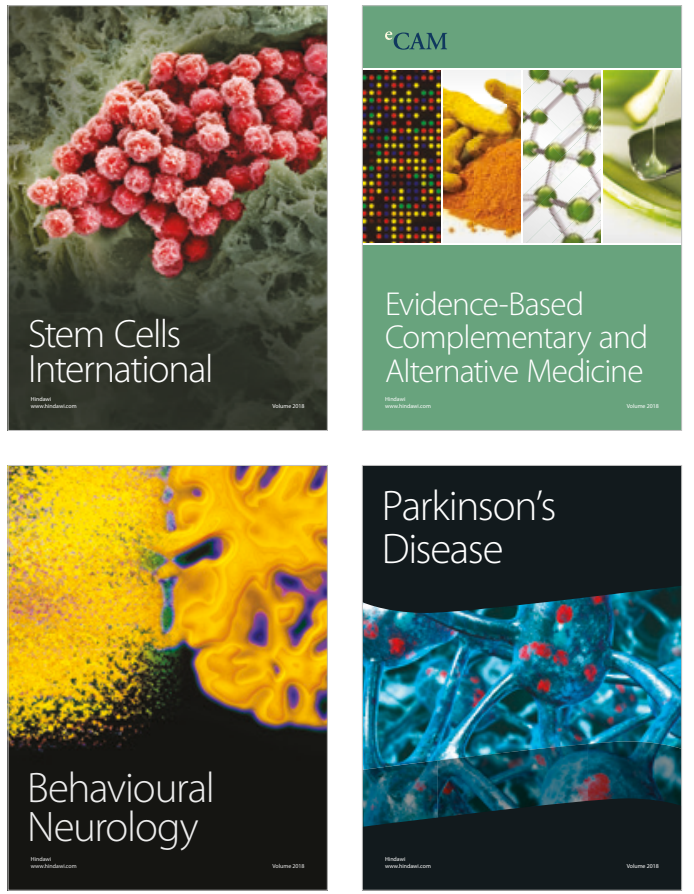

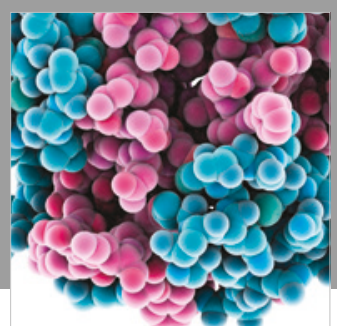

ournal of

Diabetes Research

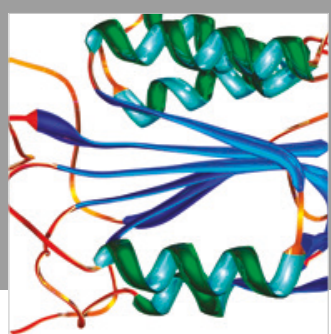

Disease Markers
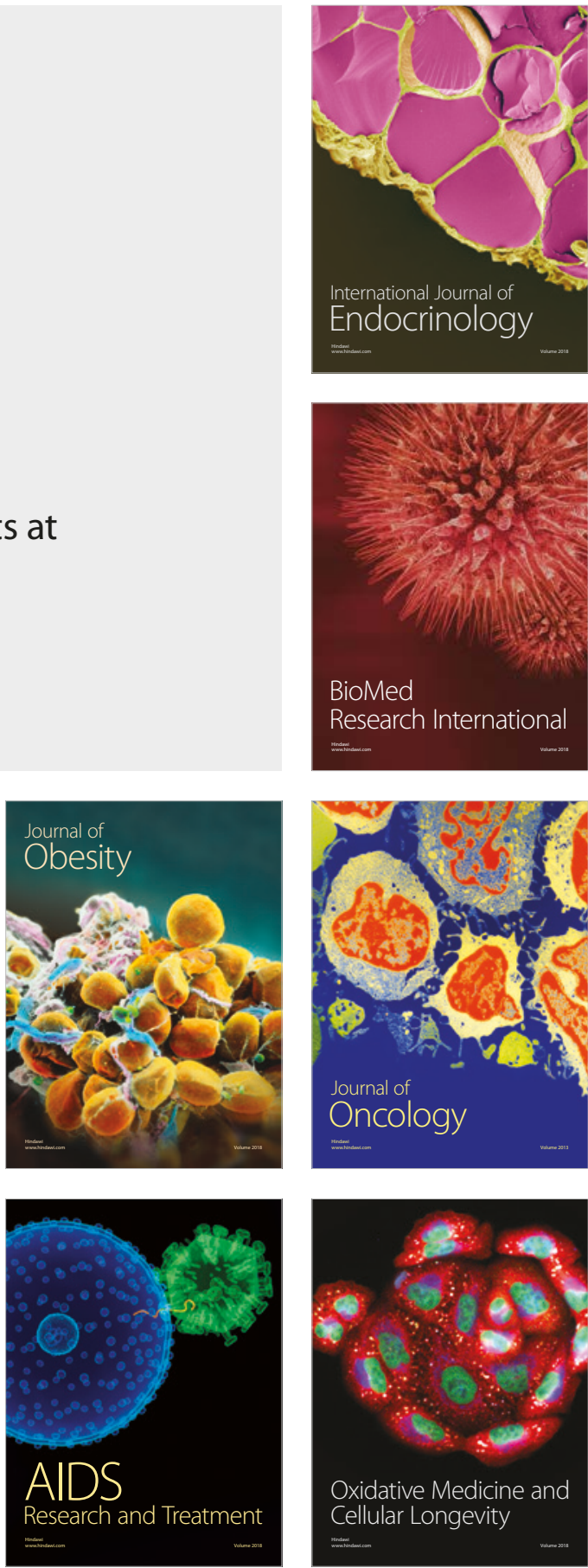